\title{
ENSAIOS DE CIRCUITOS DE PRIMEIRA ORDEM UTILIZANDO 0 LABORATÓRIO REMOTO VISIR
}

DOI: 10.37702/2175-957X.COBENGE.2021.3638

Mauro Tavares Peraça - peraca@ifsc.edu.br

Instituto Federal de Santa Catarina

Mauro Ramos 950

88020-300 - Florianópolis - SC

Luis Carlos Martinhago Schlichting - schlicht@ifsc.edu.br

Instituto Federal de Educação Ciência e Tecnologia de Santa Catarina

Avenida Mauro Ramos 950

88020-300 - Florianópolis - SC

Daniel Dezan de Bona - dezan@ifsc.edu.br

Instituto Federal de Santa Catarina

Mauro Ramos 950

88020-300 - Florianópolis - SC

Resumo: Atividades práticas são essenciais na formação de engenheiros. Assim, a busca por inovações e soluções tecnológicas que viabilizem essas atividades através de laboratórios remotos é atualmente relevante e instigante. Este trabalho apresenta a metodologia para a realização de ensaios de circuitos elétricos de primeira ordem utilizando o laboratório remoto VISIR - Virtual Instrument Systems in Reality. Inicialmente, apresenta-se uma breve contextualização do laboratório remoto VISIR, depois três abordagens de prática experimental a respeito de circuitos elétricos $R L$ e $R C$ são discutidas, sendo essas abordagens: análise fasorial, transitórios e filtros passivos. Para cada aplicação, faz-se uma breve revisão teórica, descreve-se sumariamente os roteiros dos experimentos propostos e apresenta-se os resultados obtidos a partir da utilização do laboratório remoto VISIR. Diante dos resultados, percebe-se que o laboratório remoto VISIR é uma ferramenta útil e viável para realização de práticas laboratoriais na área de circuitos elétricos, proporcionando o uso de equipamentos básicos de uma bancada de experimentos em eletrônica.

Palavras-chave: Laboratório remoto. VISIR. Experimentos. Circuitos elétricos. 


\section{ENSAIOS DE CIRCUITOS DE PRIMEIRA ORDEM UTILIZANDO O LABORATÓRIO REMOTO VISIR.}

\section{INTRODUÇÃO}

O ensino e aprendizagem de circuitos elétricos é crucial em cursos das áreas de Engenharia Elétrica e Eletrônica, tendo em vista que a modelagem matemática utilizada nas análises de circuitos servirá de base para análises e modelagens de circuitos e sistemas reais e complexos que serão enfrentados no fazer do Engenheiro. Diante disso, observa-se inúmeras tentativas e formas de estimular o aprendizado de circuitos elétricos e em muitas delas o uso de atividades de laboratório são relatadas de modo positivo.

Devido as necessidades de espaço físico e custo de equipamentos muitas instituições têm um número reduzido de laboratórios, isso limita sobremaneira o uso dos laboratórios para as unidades de circuitos elétricos, uma vez que, em muitos casos, são priorizadas atividades laboratoriais para unidades curriculares de fases mais avançadas no curso.

O uso de laboratórios remotos apresenta-se como uma alternativa viável para minimizar esses dois problemas, ou seja, permite o uso de um mesmo laboratório por diversas unidades curriculares, incluindo circuitos elétricos, demandando pouco espaço físico e recursos, sem limitações de horários, normalmente funcionando 24 horas por dia, 7 dias por semana, ampliando assim as possibilidades de acesso.

Outro aspecto que deu destaque e relevância aos laboratórios remotos foi a necessidade de interrupção de atividades presenciais de ensino devido a pandemia de COVID-19, inviabilizando o uso de laboratórios físicos tradicionais. Nesse contexto, laboratórios remotos emergem como uma grande alternativa para viabilizar atividades práticas mesmo quando as atividades de ensino são realizadas de modo "online".

O Instituto Federal de Santa Catarina dispõe de um laboratório remoto VISIR (Virtual Instrument Systems in Reality), instalado no Departamento Acadêmico de Eletrônica do Campus Florianópolis, esse laboratório tem sido bastante utilizado nas unidades curriculares de circuitos elétricos dos cursos do Instituto e de outras instituições parceiras e, nesse artigo, apresenta-se a utilização do VISIR para realização de experimentos em circuitos $\mathrm{RC}$ ou $\mathrm{RL}$ série sob três perspectivas diferentes, quais sejam: análise fasorial, transitórios e filtros passivos.

Inicialmente apresenta-se uma breve contextualização do laboratório remoto VISIR, depois as três abordagens de prática experimental são discutidas, para as quais faz-se uma breve revisão teórica, descreve-se sumariamente os roteiros dos experimentos propostos e apresenta-se os resultados obtidos a partir da utilização do laboratório remoto VISIR.

\section{LABORATÓRIO REMOTO VISIR}

O laboratório remoto VISIR (Virtual Instrument Systems in Reality) já foi apresentado e discutido em diversas publicações (ALVES, et al., 2020; GUSTAVSSON, et al., 2009; TAWFIK, et al. 2013) onde foi demonstrada a sua potencialidade e efetividade para uso na área de eletroeletrônica. Diante disso, considerando que o escopo desse artigo é a utilização do laboratório VISIR, neste tópico será feita uma breve apresentação do VISIR com o intuito apenas de situar o leitor sobre como o VISIR é composto e utilizado.

O projeto do laboratório remoto VISIR teve seu início no Instituto de Tecnologia Blekinge (BTH) - Suécia com o objetivo de disseminar o conceito de "bancada online" para experimentação (GUSTAVSSON, et al., 2009). O laboratório VISIR, consiste em um sistema que envolve hardware e software. 
O hardware é composto por uma plataforma de instrumentação PXI, da National Instruments, com módulos instrumentais como fonte de alimentação, multímetro digital, gerador de funções e osciloscópio, esses módulos são controlados através de computador. Além disso, o sistema dispõe de várias placas de componentes, sendo que cada placa comporta até dez componentes de dois terminais e dois circuitos integrados de 20 pinos cada um (TAWFIK, et al. 2013). Através de uma matriz de relés os componentes disponíveis nas placas podem ser conectados para formar os circuitos desejados, bem como conectar os pontos do circuito aos módulos de instrumentação PXI. A Figura 1 mostra o hardware do VISIR com as placas de componentes e reles sobre o módulo PXI.

Figura 1 - Hardware do VISIR.

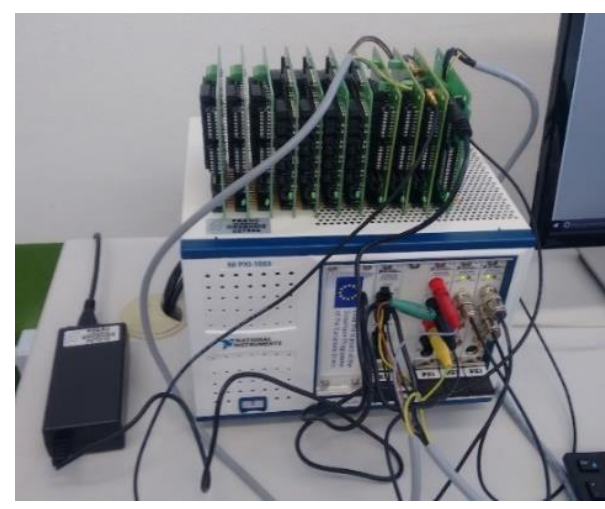

O sistema de software do VISIR é composto de várias partes, destaca-se a interface do usuário que conecta professores e estudantes ao VISIR, através dela é possível executar experimentos e verificar seus resultados reais. O VISIR dispõe de uma bancada experimental online, que conta com uma matriz de contatos e instrumentos de medida, tais como: multímetro digital, osciloscópio, fontes de alimentação DC e gerador de funções, nessa bancada o usuário poderá implementar o circuito desejado, a partir dos componentes disponíveis nas placas de componentes. Para ilustrar, apresenta-se na Figura 2 a matriz de contatos, os componentes e a instrumentação disponível no VISIR, essa imagem é um compilado de várias telas da interface do VISIR.

Figura 2- Bancada experimental online, matriz de contatos e instrumentação disponível.

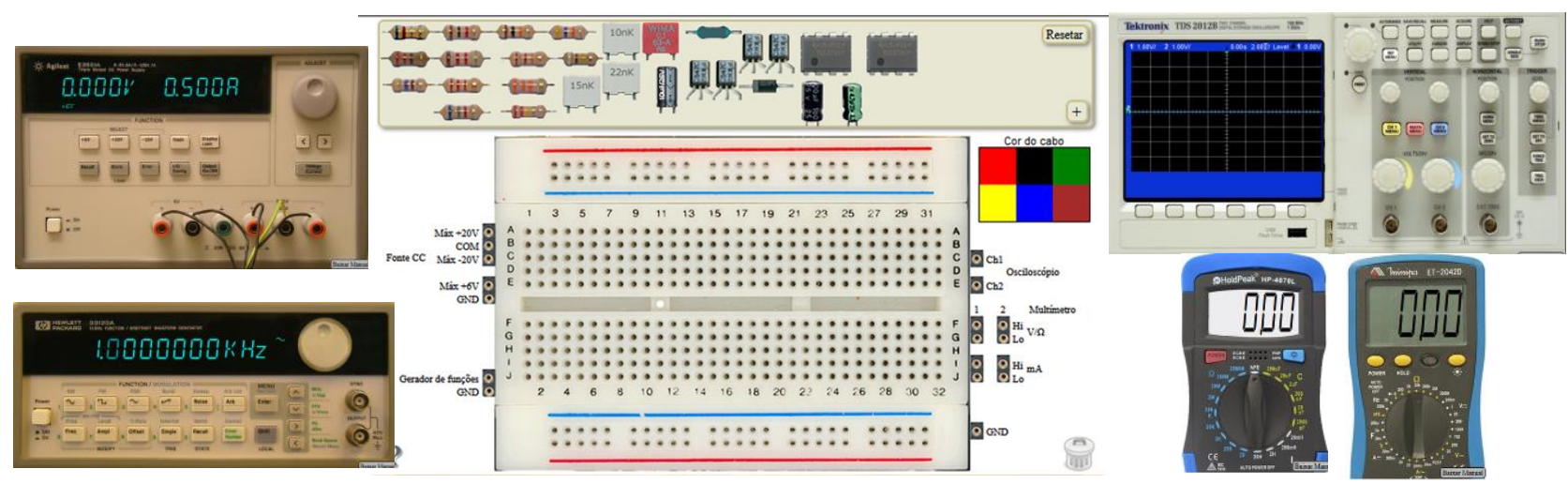

Uma vez montado o circuito, o usuário deverá acionar o botão "Realizar medição" assim o software do VISIR verifica se a montagem pode ser executada, para tanto, compara o circuito implementado com as conexões permitidas aos experimentos. Essas conexões são descritas em uma "Maxlist" previamente organizada pela equipe de suporte local do VISIR, isso garante que o experimento seja realizado de forma segura. 
Outra característica do software é que ele organiza os experimentos em fila (visto que mais de um experimento pode ser enviado ao mesmo tempo) e logo após os envia, um a um, para o equipamento a fim de realizar o experimento (TAWFIK, et al. 2013). Após realizar o experimento, o software encaminha os resultados obtidos para a interface do usuário na tela do computador, e os resultados são apresentados exatamente como num equipamento de medida real.

Considerando que o foco deste trabalho são os circuitos $R L$ e RC, apresenta-se na Figura 3 os circuitos possíveis de serem implementados, atualmente, no VISIR do IFSC. Já na Tabela 1, descreve-se os valores dos componentes disponíveis para cada circuito. As limitações de componentes e posições de ligação devem-se a forma como estes componentes estão dispostos nas placas de componentes do VISIR e as "Maxlist" disponíveis no momento.

Figura 3 - Circuitos RC e RL possíveis de serem implementados.
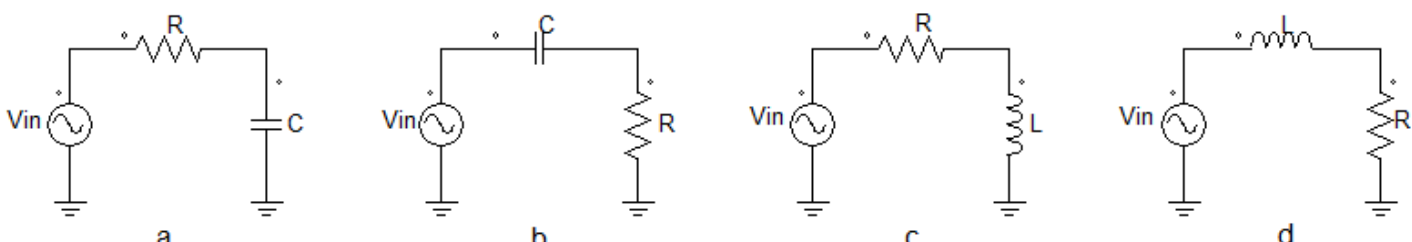

a

b

Tabela 1 - Valores dos componentes disponíveis para cada circuito.

\begin{tabular}{c|c|c|c}
\hline Circuito & Resistor $(\mathrm{k} \Omega)$ & Capacitor $(\mathrm{nF})$ & Indutor $(\mathrm{mH})$ \\
\hline $\mathrm{a}$ & 0,$47 ; 1 ; 1,2 ; 6,8$ e 22 & 15 & - \\
\hline $\mathrm{b}$ & 0,$47 ; 1 ; 1,2 ; 6,8$ e 22 & 10 e 100 & - \\
\hline $\mathrm{c}$ & 0,$47 ; 1 ; 1,2 ; 6,8$ e 22 & - & 5,6 \\
\hline $\mathrm{d}$ & 0,$47 ; 1 ; 1,2 ; 6,8$ e 22 & - & 5,6 \\
\hline
\end{tabular}

Fonte: Elaboração própria.

\section{ANÁLISE DE CIRCUITOS CA USANDO DOMÍNIO FASORIAL}

\subsection{Fundamentação teórica}

Uma das técnicas mais difundidas para a análise de circuitos em corrente alternada em regime permanente é a utilização de fasores. Segundo (ALEXANDER e SADIKU 2013), "Fasor é um número complexo que representa a amplitude e a fase de uma senoide."

A utilização de fasores facilita as operações matemáticas, substituindo funções senoidais variantes no tempo por vetores radiais girantes, esses por sua vez podem ser representados por números complexos, e assim, podemos operá-los através da álgebra aplicável aos números complexos.

Para embasar a análise teórica dos circuitos e viabilizar a comparação destes com os resultados dos experimentos realizados através do VISIR, apresenta-se resumidamente as principais expressões utilizadas em análise de circuitos CA.

Tabela 2 - Resumo das impedâncias e relações tensãocorrente no domínio temporal e fasorial.

\begin{tabular}{c|c|c|c}
\hline Elemento & $\begin{array}{c}\text { Domínio do } \\
\text { tempo }\end{array}$ & Impedância & $\begin{array}{c}\text { Domínio } \\
\text { fasorial }\end{array}$ \\
\hline $\mathrm{R}$ & $v=R \times i$ & $\mathrm{R}$ & $V=R \times I$ \\
\hline $\mathrm{L}$ & $v=L \times d i / d t$ & $X L=j \times \omega \times \mathrm{L}$ & $V=X L \times I$ \\
\hline $\mathrm{C}$ & $i=C \times d v / d t$ & $X C=1 /(j \times \omega \times \mathrm{C})$ & $V=X C \times I$ \\
\hline
\end{tabular}

Fonte: Adaptada de ALEXANDER e SADIKU 2013. 


\subsection{Roteiro do experimento}

Com o objetivo de verificar experimentalmente o comportamento de circuitos RC e/ou $\mathrm{RL}$ série em corrente alternada senoidal, utilizando o laboratório remoto VISIR, foi desenvolvido um roteiro de experimento que é disponibilizado aos discentes para a realização da atividade. Cita-se adiante os passos desse roteiro:

1. Analisar teoricamente o circuito, determinando a corrente e as tensões em cada elemento;

2. Elaborar o diagrama fasorial do circuito;

3. Simular o circuito com o intuito de validar a análise teórica, utilizando um software para simulação;

4. Implementar o circuito RC ou RL série no Laboratório VISIR;

5. Ajustar o gerador de funções para aplicar tensão senoidal com amplitude e frequência desejada;

6. Ajustar o osciloscópio (horizontal e vertical) para medir as grandezas desejadas;

7. Incluir grandezas a serem medidas, tais como: amplitude ou pico a pico, fase, frequência;

8. Executar o experimento (realizar medição);

9. Comparar os resultados experimentais com os resultados teóricos;

10. Realizar os ensaios novamente dobrando a frequência e reduzindo a frequência a metade;

11. Observar e analisar o efeito da variação da frequência sobre o comportamento do circuito;

12. Apresentar resultados através de relatório técnico.

\subsection{Resultados Experimentais}

A partir dos circuitos propostos os discentes poderão utilizar o laboratório remoto VISIR para realizar a implementação do circuito na matriz de contatos, alimentar o circuito com a tensão e frequências desejadas, através de um gerador de funções, além de realizar a medição de tensão nos elementos do circuito, através do osciloscópio, medindo as amplitudes e as defasagens das tensões.

Para esta implementação foi utilizado o circuito RC mostrado na Figura 3b, utilizando os seguintes componentes: capacitor de $10 \mathrm{nF}$ e resistor de $1 \mathrm{k} \Omega$. Cabe salientar que os componentes utilizados apresentam tolerância de 5\%.

Apresenta-se a seguir a implementação do circuito no VISIR, bem como os resultados obtidos experimentalmente, ou seja, destacaremos os passos de 4 a 8 descritos no roteiro. Vale enfatizar que é o discente que realiza a montagem do circuito, a configuração do gerador de funções e do osciloscópio, tal qual faria em um laboratório tradicional.

O circuito RC implementado na bancada experimental do VISIR pode ser visto na Figura 4. A tensão e a frequência utilizadas foram ajustadas no gerador de funções e são ilustradas Figura 5. Já na Figura 6, pode-se observar as medidas, realizadas através do osciloscópio, tensão de entrada (Ch1), tensão no resistor (Ch2) e tensão no capacitor (Math).

A Tabela 3 mostra os resultados teóricos e experimentais obtidos para o circuito $\mathrm{RC}$, assim como o erro relativo, foi dado ênfase a tensão nos elementos, considerando o módulo e a fase. Destaca-se ainda que para obtenção dos resultados teóricos foram utilizadas as expressões apresentadas na Tabela 2, e somente os resultados relevantes a comparação são apresentados diretamente na Tabela 3. 
Figura 4 - Bancada experimental - circuito RC implementado.

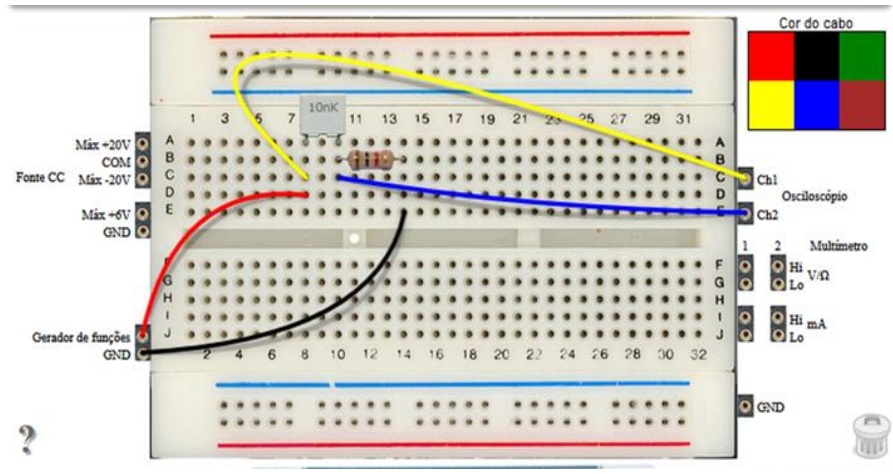

Figura 5 - Gerador de funções - frequência e tensão aplicada.
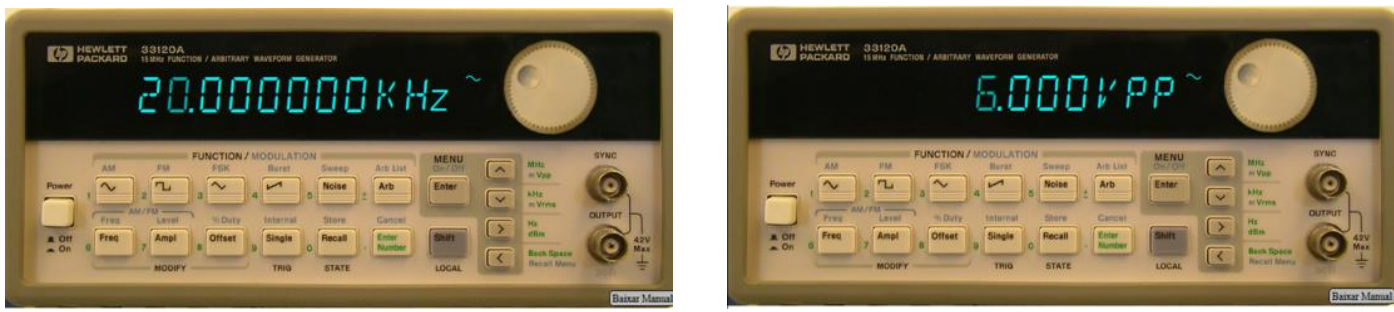

Figura 6 - Osciloscópio - Vin(Ch1), VR(Ch2), VC(Math).

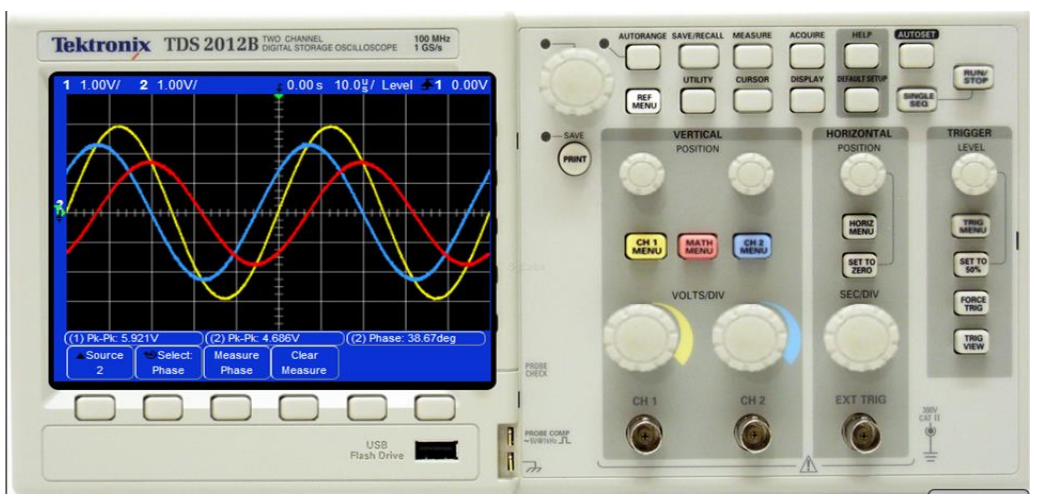

Tabela 3 - Tensões nos elementos do circuito.

\begin{tabular}{c|c|c|c|c|c|c}
\hline \multirow{2}{*}{ Elemento } & \multicolumn{3}{|c|}{ Teórico } & \multicolumn{2}{c|}{ Experimental } & \multicolumn{2}{c}{ Erro \% } \\
\cline { 2 - 7 } & Módulo $(\mathrm{Vpp})$ & Fase $\left(^{\circ}\right)$ & Módulo $(\mathrm{Vpp})$ & Fase $\left(^{\circ}\right)$ & Módulo & Fase \\
\hline Gerador & 6,00 & 0 & 5,921 & 0 & $-1,32$ & 0 \\
\hline $\mathrm{R}$ & 4,695 & 38,51 & 4,686 & 38,67 & $-0,19$ & 0,41 \\
\hline $\mathrm{C}$ & 3,736 & $-51,49$ & 3,700 & $-52,31$ & $-0,96$ & 1,60 \\
\hline
\end{tabular}

Fonte: Elaboração própria.

Para ilustrar a influência da frequência no comportamento das reatâncias e por conseguinte na corrente e nas tensões do circuito como um todo, bem como mostrar o potencial de uso do VISIR para ensaios circuitos em CA, realizou-se ainda o passo 10 do roteiro. Assim, na Figura 7a mostra-se as tensões para uma frequência de $10 \mathrm{kHz}$, já na Figura $7 \mathrm{~b}$ tem-se os resultados quando a frequência é de $40 \mathrm{kHz}$. Destaca-se que a base de tempo do osciloscópio é diferente em cada aquisição e que esse ajuste deve ser feito pelo usuário. 
Figura 7 - Osciloscópio - Vin(Ch1), VR(Ch2), VC(Math).

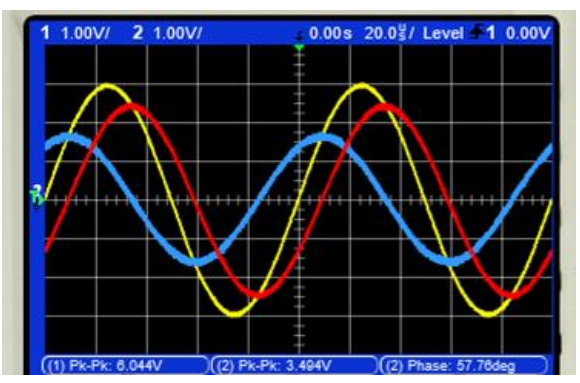

b

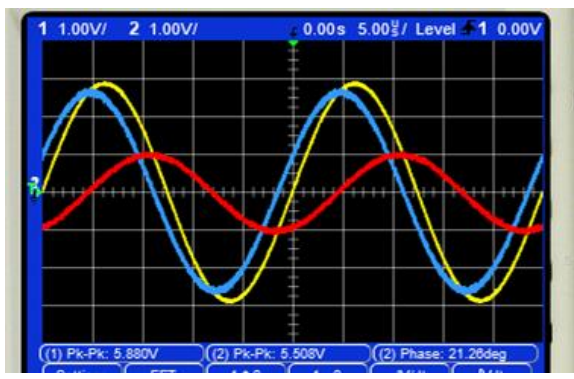

\subsection{Fundamentação teórica}

A análise de circuitos simples compostos por resistor e indutor ou por resistor e capacitor resultam em equações diferenciais de primeira ordem, por isso eles são conhecidos como circuitos de primeira ordem (ALEXANDER e SADIKU 2013). A solução das equações diferenciais que regem esses circuitos depende basicamente de três fatores, quais sejam: condições iniciais, constante de tempo e condições finais.

Para circuitos RL, a condição inicial e a condição final são dadas, respectivamente, pela corrente que passa no indutor no instante inicial de observação e pela corrente de regime permanente, ou seja, a corrente que circula após o transitório. Já nos circuitos $\mathrm{RC}$, essas condições são determinadas pela tensão do capacitor.

Considerando que a energia armazenada no indutor ou capacitor não pode ser alterada instantaneamente, nota-se que todo circuito $\mathrm{RL}$ ou $\mathrm{RC}$ demora um tempo para alterar da condição inicial para a condição final, normalmente considera-se que o tempo necessário para tanto são cinco constantes de tempo $(5 \tau)$. Para circuitos sem fonte, segundo (ALEXANDER e SADIKU 2013), "a constante de tempo de um circuito é o tempo necessário para a resposta de decaimento a um fator igual a $1 /$ e ou a $36,8 \%$ do seu valor inicial', por extensão, pode-se concluir que após cinco constantes de tempo a resposta será $1 / e^{5}$, logo, a saída será $0,67 \%$ do valor inicial.

Para embasar a análise teórica dos circuitos e viabilizar a comparação destes com os resultados dos experimentos realizados através do VISIR, apresenta-se resumidamente na Tabela 4 as principais expressões utilizadas para circuitos de primeira ordem.

Tabela 4 - Resumo das equações para circuitos RL e RC.

\begin{tabular}{c|c|c|c}
\hline Circuito & Tensão & Corrente & $\tau$ \\
\hline $\mathrm{RL}$ & $v_{L}=L \times d i / d t$ & $i_{L}(t)=i(\infty)+[i(0)-i(\infty)] \times e^{-t / \tau}$ & $\tau=L / R$ \\
\hline $\mathrm{RC}$ & $v_{C}(t)=v(\infty)+[v(0)-v(\infty)] \times e^{-t / \tau}$ & $i_{C}=C \times d v / d t$ & $\tau=R \times C$ \\
\hline \multicolumn{4}{r}{ Fonte: Adaptada de ALEXANDER e SADIKU 2013. }
\end{tabular}

\subsection{Roteiro do experimento}

Com o objetivo de verificar experimentalmente o comportamento transitório de circuitos $\mathrm{RC}$ e/ou $\mathrm{RL}$ série utilizando o laboratório remoto VISIR foi desenvolvido um roteiro de experimento que é disponibilizado aos discentes para a realização da atividade. Cita-se adiante os passos desse roteiro:

1. Analisar teoricamente o circuito, determinando a corrente e as tensões em cada elemento;

2. Elaborar um esboço do transitório ( $\mathrm{V}$ e I); 
3. Simular o circuito com o intuito de validar a análise teórica, utilizando um software para simulação;

4. Implementar o circuito RC ou RL série no Laboratório VISIR;

5. Ajustar o gerador de funções para aplicar tensão quadrada com amplitude, offset e frequência desejada;

6. Ajustar o osciloscópio (horizontal e vertical) para medir as grandezas desejadas;

7. Incluir grandezas a serem medidas, tais como: pico a pico, frequência;

8. Executar o experimento (realizar medição);

9. Comparar os resultados experimentais com os resultados teóricos;

10. Apresentar resultados através de relatório técnico.

\subsection{Resultados Experimentais}

Utilizando o laboratório remoto VISIR, baseados nos circuitos propostos, os discentes poderão realizar a implementação do circuito na matriz de contatos, alimentar o circuito com a forma de onda, amplitude e frequências desejadas, através de um gerador de funções, além de realizar a medição de tensão nos elementos do circuito, através do osciloscópio. Na Figura 3 tem-se os circuitos disponíveis para implementação.

Para esta implementação foi utilizado o circuito RL mostrado na Figura 3d, utilizando os seguintes componentes: indutor de $5,6 \mathrm{mH}$ e resistor de $1 \mathrm{k} \Omega$. De modo similar ao laboratório tradicional, definidos os componentes, o discente realiza a montagem do circuito, a configuração do gerador de funções e do osciloscópio, desse modo, enfatizaremos adiante os passos de 4 a 8 descritos no roteiro.

Vale destacar que ao propor o uso de uma onda quadrada na entrada do circuito, ocorrerão transitórios de carga e descarga e, portanto, para poder observar ambos, o período da tensão entrada deverá ser de pelo menos $10 \tau, 5 \tau$ para carga e $5 \tau$ para descarga. Assim, a frequência aplicada ao circuito deve ser no máximo $1 / 10 \tau$.

O circuito implementado na bancada experimental do VISIR para observar o transitório do circuito $R L$ pode ser visto na Figura 8 . Através do gerador de funções faz-se os ajustes da tensão, offset e da frequência utilizadas, optou-se por um período de $40 \mu \mathrm{s}$ para facilitar a visualização no osciloscópio.

Figura 8 - Bancada experimental - circuito RL implementado.

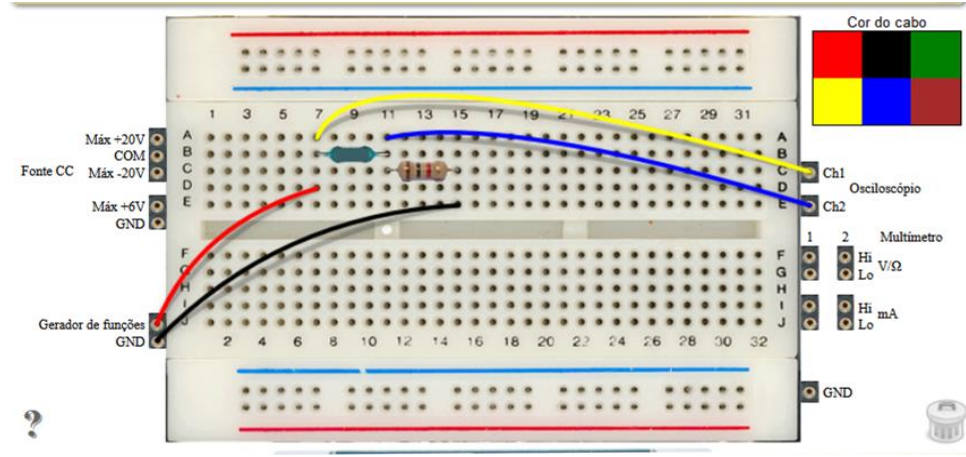

$\mathrm{Na}$ Figura 9, pode-se observar as medidas, realizadas através do osciloscópio, tensão de entrada (Ch1) e tensão no resistor (Ch2), destaca-se que a tensão no resistor é proporcional a corrente que circula no circuito, considerando que o resistor é de $1 \mathrm{k} \Omega$, a corrente corresponde a um milésimo da tensão medida. Na Figura 10, pode-se observar a tensão no indutor através da função matemática disponível no osciloscópio (Ch1-Ch2), como a corrente no indutor não muda instantaneamente a tensão no resistor também não 
se altera instantaneamente, logo, quando ocorrem os transitórios toda a variação da tensão é aplicada inicialmente no indutor, como se vê claramente na aquisição.

Figura 9 - Osciloscópio - Vin(Ch1), VR(Ch2).

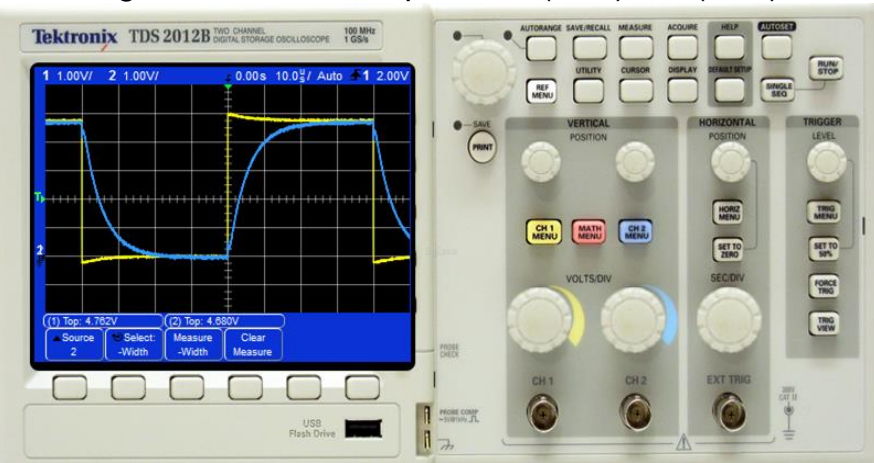

Figura 10 - Osciloscópio - Vin(Ch1), VR(Ch2), VL(Math).

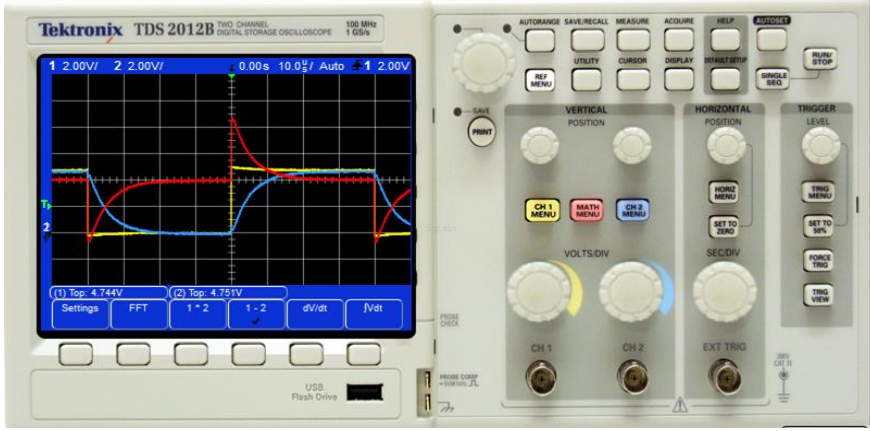

A Figura 11 mostra os resultados teóricos para o circuito $\mathrm{RL}$ implementado. Para obtenção dos resultados teóricos foram utilizadas as expressões apresentadas na Tabela 4 e, a partir delas, traçados gráficos para as tensões no resistor e no indutor para o transitório de descarga, à esquerda, e para o transitório de carga, à direita. Pode-se notar a semelhança das curvas teóricas com aquelas obtidas experimentalmente. Nas curvas teóricas são indicadas as tensões no resistor $10 \mu$ s após os transitórios e se observarmos esses mesmos pontos no osciloscópio podemos verificar que os resultados experimentais são muito semelhantes aos teóricos.

Figura 11 - Tensão no resistor e no indutor.
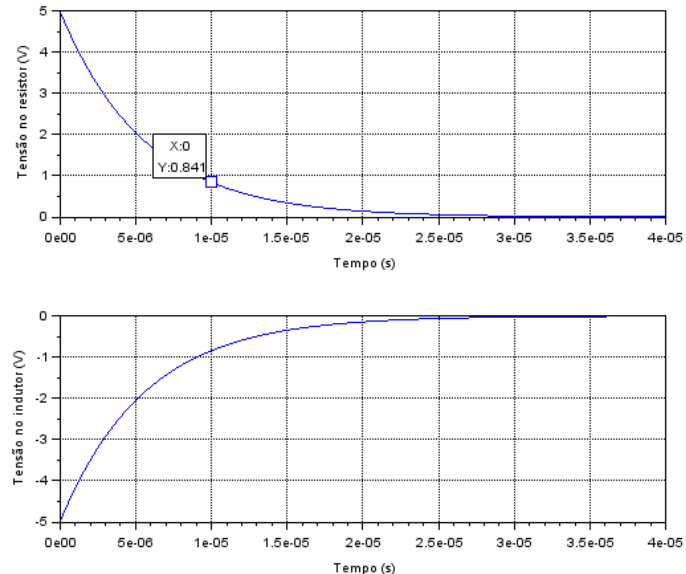
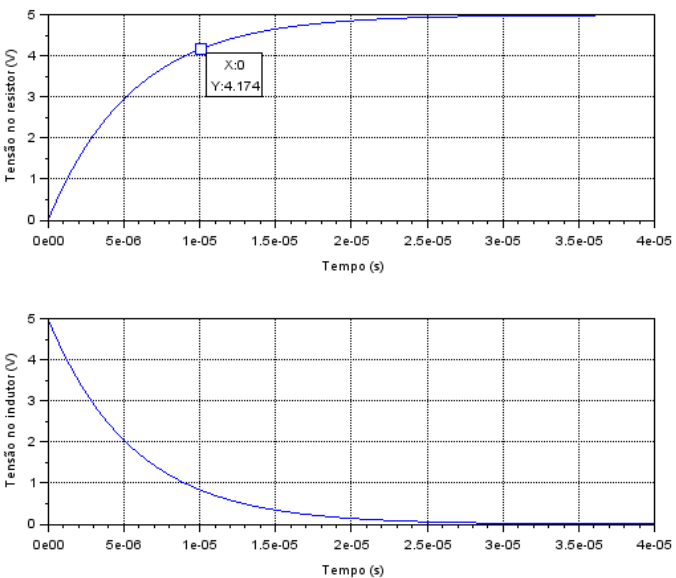


\section{$5 \quad$ ANÁLISE DE FILTROS DE PRIMEIRA ORDEM}

\subsection{Fundamentação teórica}

Circuitos elétricos podem ser excitados por frequências diferentes e muitas vezes apresentam comportamentos diferentes em função da frequência, diante disso, é de suma importância para análise de circuitos a compreensão da resposta em frequência. Segundo (ALEXANDER e SADIKU 2013), "Resposta de frequência de um circuito é a variação em seu comportamento em virtude da mudança na frequência dos sinais."

Para observar-se a resposta em frequência de circuitos, dentre as ferramentas mais utilizadas destaca-se a função de transferência e os diagramas de Bode. A Função de transferência relaciona a grandeza de saída com a grandeza de entrada em função da frequência, em circuitos elétricos são muito utilizados o ganho de tensão ou corrente. Ainda, segundo (ALEXANDER e SADIKU 2013), os "Gráficos de Bode são gráficos semilogarítmicos da magnitude (em decibéis) e da fase (em graus) de uma função de transferência versus frequência", ou seja, são uma representação gráfica da função de transferência.

Filtros passivos são circuitos formados apenas por elementos passivos $R, L$ e $C$. De acordo com (ALEXANDER e SADIKU 2013), "Filtro é um circuito projetado para deixar passar sinais com frequências desejadas e rejeitar ou atenuar outros." No caso de filtros de primeira ordem, circuitos simples compostos por um $\mathrm{R}$ e um $\mathrm{L}$, ou por um $\mathrm{R}$ e um $\mathrm{C}$ podem realizar as funções de filtros passa-baixas ou passa-altas, dependendo da forma de implementação do circuito. Nesses filtros, considera-se como frequência de corte $(\omega c)$, a frequência na qual a tensão de saída é raiz de duas vezes menor que a de entrada.

Diante disso, para embasar a análise teórica dos filtros passivos de primeira ordem e viabilizar a comparação destes com os resultados dos experimentos realizados através do VISIR, apresenta-se resumidamente as principais funções de transferência utilizadas nesses filtros.

Tabela 5 - Resumo das funções de transferência de filtros de primeira ordem.

\begin{tabular}{c|c|c|c}
\hline Circuito & Passa-baixas & Passa-altas & $\omega c$ \\
\hline $\mathrm{RL}$ & $H(\omega)=1 /(1+j \omega L / R)$ & $H(\omega)=(j \omega L / R) /(1+j \omega L / R)$ & $\omega c=R / L$ \\
\hline $\mathrm{RC}$ & $H(\omega)=1 /(1+j \omega R C)$ & $H(\omega)=(j \omega R C) /(1+j \omega R C$ & $\omega c=1 / R C$ \\
\hline
\end{tabular}

Fonte: Fonte: Adaptada de ALEXANDER e SADIKU 2013

\subsection{Roteiro do experimento}

Com o objetivo de verificar experimentalmente o comportamento de filtros passivos de primeira ordem utilizando o laboratório remoto VISIR foi desenvolvido um roteiro de experimento que é disponibilizado aos discentes para a realização da atividade. Cita-se adiante os passos desse roteiro:

1. Analisar teoricamente o circuito, determinando a função de transferência;

2. Traçar o diagrama de Bode da função de transferência;

3. Simular o circuito em diferentes frequências com o intuito de validar a análise teórica, utilizando um software para simulação;

4. Implementar o circuito $\mathrm{RC}$ ou $\mathrm{RL}$ série para obter o filtro desejado no Laboratório VISIR;

5. Ajustar o gerador de funções para aplicar tensão senoidal com amplitude e frequência desejada;

6. Ajustar o osciloscópio (horizontal e vertical) para medir as grandezas desejadas;

7. Incluir grandezas a serem medidas, tais como: RMS ou pico a pico, fase, frequência;

8. Executar o experimento (realizar medição); 
9. Comparar os resultados experimentais com os resultados teóricos;

10. Realizar novos ensaios em diversas frequências (repetir passos 5, 6 e 8);

11. Observar e analisar o efeito da variação da frequência sobre o comportamento do circuito;

12. Apresentar resultados através de relatório técnico.

\subsection{Resultados Experimentais}

Utilizando o laboratório remoto VISIR os estudantes implementarão os circuitos propostos, poderão excitar o circuito com a tensão senoidal, com as amplitudes e as frequências desejadas, através de um gerador de funções, além de realizar as medições de tensão nos elementos do circuito, através do osciloscópio, medindo as amplitudes e as defasagens das tensões. Os circuitos disponíveis $\mathrm{p} /$ implementação são os mostrados na Figura 3, já os componentes disponíveis estão descritos na Tabela 1.

Para essa implementação foi utilizado o circuito RC mostrado na Figura 3a, utilizando os seguintes componentes: capacitor de $15 \mathrm{nF}$ e resistor de $6,8 \mathrm{k} \Omega$, com isso a frequência de corte do filtro será $9804 \mathrm{rad} / \mathrm{s}$, ou seja, $1560 \mathrm{~Hz}$. Definidos os componentes, do mesmo modo que em um laboratório tradicional, o discente realiza a montagem e os ensaios no circuito.

Apresenta-se a seguir a implementação do circuito no VISIR, bem como os resultados obtidos experimentalmente, ou seja, destacaremos os passos de 4 a 8 descritos no roteiro. Nessa etapa o discente é o ator principal da atividade, ele realiza a montagem do circuito, ajusta amplitude e frequência no gerador de funções e prepara o osciloscópio (horizontal e vertical) para visualizar as tensões medidas, do mesmo modo que seria necessário em um laboratório convencional.

O circuito implementado no VISIR, para o filtro passa-baixa RC, pode ser visto na Figura 12. Por tratar-se de filtros, serão realizadas medições em várias frequências. $\mathrm{Na}$ Figura 13, pode-se observar as medidas, realizadas através do osciloscópio, tensões de entrada (Vin) e de saída (Vout). Na Figura 13a pode-se observar os resultados do circuito operando com frequência uma década abaixo da frequência de corte, já na Figura 13b o filtro está excitado na própria frequência de corte, enquanto na Figura 13c trabalha uma década acima.

Nota-se claramente na Figura 13 a operação do filtro passa-baixas uma vez que na primeira imagem a tensão de saída é praticamente igual à da entrada, na segunda a tensão foi reduzida em $\sqrt{2}$, já na terceira observa-se uma atenuação de $20 \mathrm{~dB}$, ou seja, a saída é aproximadamente um décimo da entrada.

Figura 12 - Bancada experimental - filtro RC implementado.

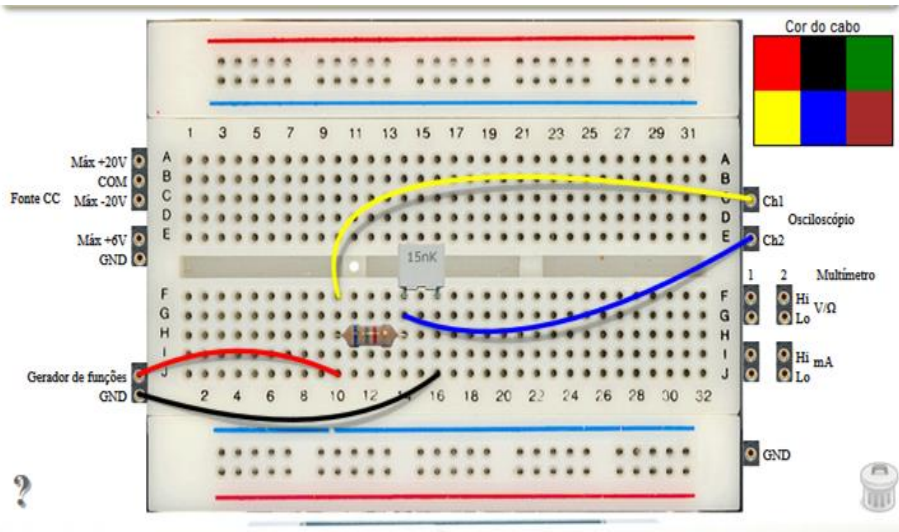


Figura 13 - Osciloscópio - Vin(Ch1), Vout(Ch2).
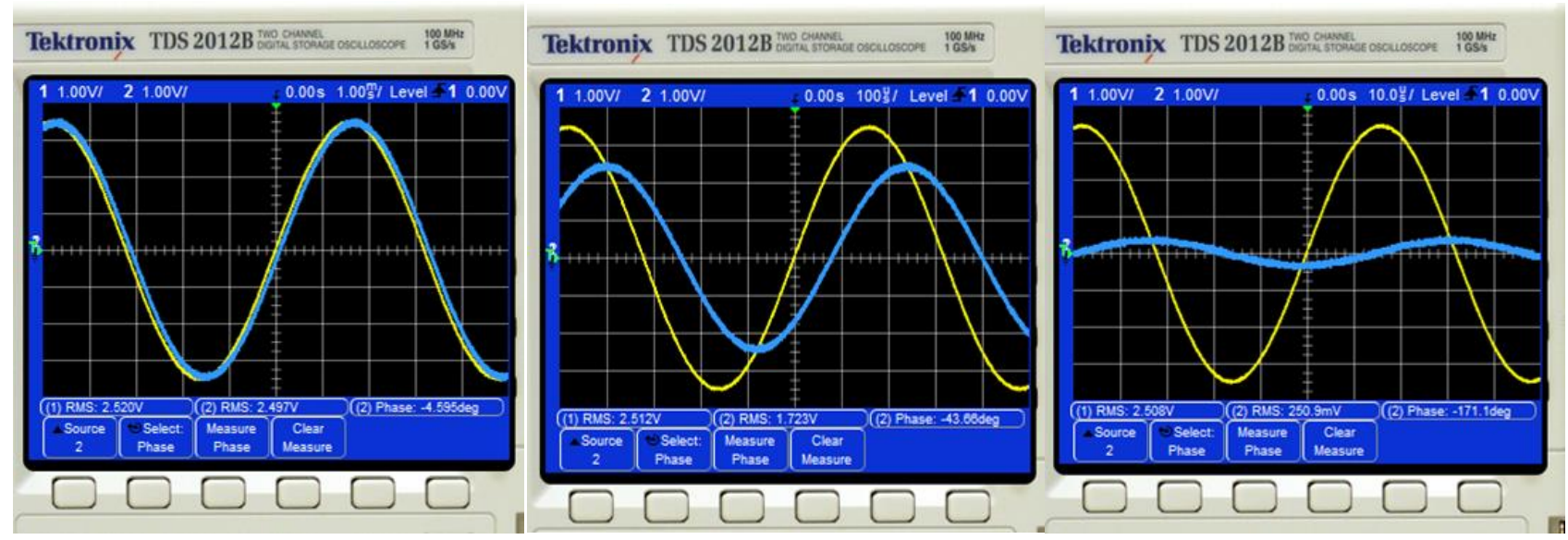

A Tabela 6 mostra os resultados teóricos e experimentais obtidos para o circuito do filtro passa-baixas implementado, assim, tem-se o ganho, o ganho em dB e o erro relativo. Destaca-se ainda que para obtenção dos resultados teóricos foram utilizadas as expressões apresentadas na Tabela 5, aplicando os valores nominais dos componentes $R$ e C utilizados, e somente os resultados relevantes a comparação são apresentados diretamente na Tabela 6.

\begin{tabular}{c|c|c|c|c|c}
\multicolumn{7}{c}{ Tabela 6 - Ganhos teórico e experimental. } \\
\cline { 2 - 6 } Frequência(Hz) & \multicolumn{2}{|c|}{ Teórico } & \multicolumn{2}{c|}{ Experimental } & Erro \% \\
\cline { 2 - 6 } & Ganho & GdB & Ganho & GdB & Ganho \\
\hline 156 & 0,995 & $-0,0432$ & 0,991 & $-0,0796$ & $-0,42$ \\
\hline 1560 & 0,707 & $-3,009$ & 0,686 & $-3,275$ & $-3,01$ \\
\hline 15600 & 0,0995 & $-20,041$ & 0,100 & $-19,996$ & 0,52 \\
\hline
\end{tabular}

Fonte Elaboração própria.

\section{Considerações Finais}

Este artigo, reconhecendo a necessidade da utilização de experimentos práticos no processo ensino e aprendizagem em cursos de graduação em engenharia, apresenta roteiros de experimentos em circuitos de primeira ordem, abordando três ênfases, quais sejam: análise fasorial, transitórios e filtros.

Diante da inexistência de um laboratório para realização destes experimentos ou da impossibilidade de realização presencial devido ao momento atual (COVID 19) o artigo demonstra que o laboratório remoto VISIR apresenta-se como uma ferramenta que viabiliza a realização destes experimentos.

Percebe-se pelo roteiro e pelos resultados apresentados que o VISIR além de viabilizar os experimentos, possibilita que o aluno fixe conteúdos e aprenda a trabalhar com equipamentos básicos de uma bancada de experimentos em eletrônica.

\section{Agradecimentos}

Os autores deste artigo agradecem ao apoio do Programa Erasmus+ e ao Instituto Federal de Santa Catarina que, via seus editais internos, possibilita a aplicação, manutenção e expansão do sistema VISIR.

\section{REFERÊNCIAS}


About VISIR. Disponível em: https://visir.florianopolis.ifsc.edu.br. Acesso em: 06 mai. 2021.

ALVES, Gustavo Ribeiro et al. Resultados e Extensões do Projeto VISIR+ no Brasil: Um Olhar Internacional. Brasília: Editora ABENGE. 2020.

ALEXANDER, Charles K.; SADIKU, Matthew N. O. Fundamentos de Circuitos Elétricos 5 ed. Porto Alegre AMGH, 2013. 874 p.

BRANCO, Matheus V.; et al. Aspectos de Diferenciação entre Laboratórios Remotos e Simuladores. In: XLV Congresso Brasileiro de Educação em Engenharia, 2017, Joinville. Anais. Joinville, 2017.

GUSTAVSSON, I. et al. On Objectives of Instrucional Laboratories, Individual Assessment, and Use of Collaborative Remote Laboratories. IEEE Transactions on Learning Technologies, v.2, ํㅜㄴ, p.263-273, oct.-dez. 2009.

PERAÇA, M. T. et al. Ensaios para Caracterização de Quadripolos Utilizando o Laboratório Remoto VISIR. In: XLVI Congresso Brasileiro de Educação em Engenharia, 2018, Salvador. Anais. Salvador, 2018.

ROQUE, Gabriela R. et al. Utilização do Laboratório Remoto VISIR como Recurso Educacional num Curso de Engenharia Mecatrônica. In: XLV Congresso Brasileiro de Educação em Engenharia, 2017, Joinville. Anais. Joinville, 2017.

TAVARES, Isabelli Sasdelli et al. Caracterização de Erros Experimentais em Circuitos Eletrônicos no Laboratório Remoto VISIR+. In: XLVIII Congresso Brasileiro de Educação em Engenharia, 2020, Caxias do Sul. Anais. Joinville, 2020.

TAWFIK, M. et al. Virtual Instruments Systems in Reality (VISIR) for Remote Wiring and Measurement of Electronic Circuits on Breadboard. IEEE Transactions on Learning Technologies, v.6, no 1, p.60-72, 2013.

\section{FIRST-ORDER CIRCUITS ANALYSIS USING THE VISIR REMOTE LABORATORY}

Abstract: Practical activities are essential in the education of engineers. Due to that, the search for innovations and technological solutions that make education possible through remote laboratories is relevant and challenging. This paper means to present the methodology for conducting first-order electrical circuit tests using the remote VISIR - Virtual Instrument Systems in Reality - laboratory. Initially is presented a short contextualization of the remote VISIR laboratory then three approaches of experimental practice regarding electrical circuits RL and RC are discusses which are phasor analysis, transients and passive filters. For each application, a short theoretical review and a guide for the proposed experiments are described. After that, the results obtained from the use of the remote VISIR laboratory are presented. Due to the results achieved it can be concluded that the VISIR remote laboratory is an useful and viable tool for carrying out laboratory practices in the area of electrical circuits as it provide the use of equipment from an electronics experiment bench.

Keywords: Remote laboratory. VISIR. Experiments. Electric circuits. 